\title{
Adaptability of Closed Loop During Labor, Delivery, and Postpartum: A Secondary Analysis of Data from Two Randomized Crossover Trials in Type 1 Diabetes Pregnancy
}

\author{
Zoe A. Stewart, MD, ${ }^{1,2, *}$ Jennifer M. Yamamoto, MD, ${ }^{3, *}$ Malgorzata E. Wilinska, PhD, \\ Sarah Hartnell, $\mathrm{BSc}^{2}$ Conor Farrington, $\mathrm{PhD}^{4}$ Roman Hovorka, PhD, and Helen R. Murphy, MD ${ }^{1,2,5}$
}

\begin{abstract}
Tight glucose control during labor and delivery is recommended for pregnant women with type 1 diabetes. This can be challenging to achieve using the current treatment modalities. The automated nature of closed loop and its ability to adapt to real-time glucose levels make it well suited for use during labor, delivery, and the immediate postpartum period. We report observational data of participants from two randomized crossover trials who chose to continue using closed loop during labor, delivery, and postpartum. Labor was defined as the $24 \mathrm{~h}$ before delivery and postpartum as the $48 \mathrm{~h}$ after delivery. The glucose target range during pregnancy was $3.5-7.8 \mathrm{mmol} / \mathrm{L}(63-140 \mathrm{mg} / \mathrm{dL})$ and $3.9-10 \mathrm{mmol} / \mathrm{L}(70-180 \mathrm{mg} / \mathrm{dL})$ after delivery. Twenty-seven $(84.4 \%)$ of the potential 32 trial participants used closed loop through labor, delivery, and postpartum. Use of closed loop was associated with $82.0 \%$ (interquartile range [IQR] 49.3, 93.0) time-in-target range during labor and delivery and a mean glucose of $6.9 \pm 1.4 \mathrm{mmol} / \mathrm{L}(124 \pm 25 \mathrm{mg} / \mathrm{dL})$. Closed loop performed well throughout vaginal, elective, and emergency cesarean section deliveries. Postpartum, women spent 83.3\% (IQR 75.2, 94.6) time-intarget range $(3.9-10.0 \mathrm{mmol} / \mathrm{L}[70-180 \mathrm{mg} / \mathrm{dL}])$, with a mean glucose of $7.2 \pm 1.4 \mathrm{mmol} / \mathrm{L}(130 \pm 25 \mathrm{mg} / \mathrm{dL})$. There was no difference in maternal glucose concentration between mothers of infants with and without neonatal hypoglycemia $(6.9 \pm 1.6 \mathrm{mmol} / \mathrm{L}$ and $6.8 \pm 1.1 \mathrm{mmol} / \mathrm{L}[124 \pm 29 \mathrm{mg} / \mathrm{dL}$ and $122 \pm 20 \mathrm{mg} / \mathrm{dL}] \mathrm{respec}-$ tively; $P=0.84$ ). Automated closed-loop insulin delivery is feasible during hospital admissions for labor, delivery, and postpartum. Larger scale studies are needed to evaluate its efficacy compared with current clinical approaches as well as understand how women and healthcare providers will adopt this technology.
\end{abstract}

Keywords: Diabetes, Pregnancy, Closed loop, Insulin, Labor and delivery.

\section{Background}

A CHIEVING OPTIMAL GLYCEMIC control throughout pregnancy remains exceptionally demanding for women with type 1 diabetes (T1D). ${ }^{1}$ Peripartum maternal hyperglycemia is frequently, but inconsistently, associated with a higher risk of neonatal hypoglycemia. ${ }^{2}$ Thus, tight glucose control during labor and delivery is generally recommended. ${ }^{3-5}$ The physiological stresses and dramatic changes in insulin doses during labor and delivery, supervised by obstetric ward staff with limited diabetes expertise, represent a unique challenge.

\footnotetext{
${ }^{1}$ Wellcome Trust-Medical Research Council Institute of Metabolic Science, University of Cambridge, Cambridge, United Kingdom.

${ }^{2}$ Wolfson Diabetes and Endocrine Clinic, Cambridge University Hospitals NHS Foundation Trust, Cambridge, United Kingdom.

${ }^{3}$ Department of Medicine, University of Calgary, Calgary, Alberta, Canada.

${ }^{4}$ Department of Public Health and Primary Care, THIS Institute, University of Cambridge, Cambridge, United Kingdom.

${ }^{5}$ Department of Medicine, Norwich Medical School, University of East Anglia, Norwich, United Kingdom.

*These authors contributed equally to this work.

This has been submitted in abstract form to the American Diabetes Association 78th Scientific Sessions, June 2018, Orlando, FL.

(C) Zoe A. Stewart, et al., 2018; Published by Mary Ann Liebert, Inc. This Open Access article is distributed under the terms of the Creative Commons Attribution Noncommercial License (http://creativecommons.org/licenses/by-nc/4.0/) which permits any noncommercial use, distribution, and reproduction in any medium, provided the original author(s) and the source are credited.
} 
The changes in cortisol, increased glucose utilization by uterine and skeletal muscles, and the dramatic decrease in insulin resistance immediately after delivery make tight control difficult to achieve. ${ }^{6}$ Even with the recommended hourly capillary glucose monitoring, hypo- and hyperglycemic excursions are common and frequent insulin dose adjustments are required. ${ }^{4,7}$

Variable rate intravenous insulin infusion (VRIII) and continuous subcutaneous insulin infusion (CSII) are the most commonly used modes of insulin delivery during labor and delivery. Women on multiple daily injections (MDI) are generally treated with VRIII. Those on CSII are often transitioned to VRIII, however, if women are comfortable self-managing their CSII and can maintain the target glucose concentration, insulin pump therapy can be safely continued. ${ }^{4,5,7}$ Self-management using CSII is less invasive and may provide better glycemic control than VRIII during this period. ${ }^{7}$

Furthermore, some women report feeling vulnerable when the ability to control their own glucose is taken away from them and is instead "in the hands" of less experienced obstetric ward staff. ${ }^{8}$ For women who continue managing their own insulin delivery, the burden of frequent monitoring and insulin dose adjustments can be stressful. ${ }^{9}$

A hybrid closed-loop system uses a continuous glucose monitor (CGM), CSII, and computer algorithm to provide automated glucose-responsive insulin delivery. In pregnancy, it has been shown to improve overnight time in target and safely maintain effective glucose control with less hypoglycemia compared with sensor-augmented pump (SAP) therapy. ${ }^{10,11}$ Its automated nature with minimal obstetric staff and user input makes it well suited for use during labor, delivery, and the immediate postpartum period.

Two randomized crossover trials of home closed loop in pregnancy have been performed. The first examined the efficacy of overnight closed loop and demonstrated better glucose control overnight when compared with SAP therapy. ${ }^{10}$ The second trial examined day and night use of closed loop in pregnancy and found comparable glucose control measured by time in target with less hypoglycemia. ${ }^{11} \mathrm{We}$ report on data collected from these randomized crossover trials examining in pregnancy. ${ }^{10,11}$

The aim of this study is to evaluate the feasibility and safety of closed loop for maintaining glucose control in women with T1D during labor, delivery, and the immediate postpartum period.

\section{Methods}

\section{Participants}

Both trials recruited pregnant women 18 to 45 years of age with T1D duration of at least 12 months and HbA1c of $6.5 \%-10 \%(48-86 \mathrm{mmol} / \mathrm{mol})$. Women were randomized to 4 weeks of closed loop and 4 weeks of SAP therapy, separated by a 1-2-week washout period. After completing both arms, women could choose to continue using SAP, closed loop, or their preferred CGM/CSII/MDI combination. Women who used closed loop for labor and delivery could continue it until their discharge from hospital during the overnight study ${ }^{10}$ or for up to 6 weeks postpartum in the day-and-night study. ${ }^{11}$

The trial protocols were approved by Regional Ethics Committees. As both protocols allowed for use of data throughout pregnancy, no separate consent was required.

\section{Study design}

This was an observational study of all women included in the trials described who chose to use closed loop during labor, delivery, and the immediate postpartum period.

"Labor" was defined as the $24 \mathrm{~h}$ prior to delivery and "the immediate postpartum period" as the $48 \mathrm{~h}$ after delivery. Glucose control was measured by CGM (FreeStyle Navigator 2; Abbott Diabetes Care, Alameda, CA), insulin delivery was through a DANA-R pump (Diabecare, Sooil, Seoul, South Korea) and the closed-loop systems used were the Florence D2W and Florence D2A (both University of Cambridge, Cambridge, UK). The control algorithm was on a tablet PC for the former and on an Android phone for the latter.

The glucose target range during pregnancy was 3.5$7.8 \mathrm{mmol} / \mathrm{L}(63-140 \mathrm{mg} / \mathrm{dL})$. These targets were changed to $3.9-10 \mathrm{mmol} / \mathrm{L}(70-180 \mathrm{mg} / \mathrm{dL})$ immediately after delivery. There were no cues or changes in programming to announce the onset of labor and/or delivery. Women were administered preprandial and any additional correction boluses through their insulin pump or the Android mobile phone if they were using the Florence D2A system.

Women were advised to change their insulin pump settings and carbohydrate ratios as soon as possible after delivery, with postnatal glucose targets of $6-10 \mathrm{mmol} / \mathrm{L}(108-180 \mathrm{mg} /$ dL). Participants were instructed not to bolus for their first light meal and only to correct high glucose concentrations, defined as $\geq 12 \mathrm{mmol} / \mathrm{L}(216 \mathrm{mg} / \mathrm{dL})$.

Glucose control was assessed by mode of delivery (vaginal delivery, emergency, or elective cesarean section). Maternal hypoglycemic events during intrapartum and postpartum periods were defined as events lasting $>20$ min with a CGM glucose of $<3.9 \mathrm{mmol} / \mathrm{L}(70 \mathrm{mg} / \mathrm{dL})$ (level 1) and $<3.5 \mathrm{mmol} / \mathrm{L}$ $(63 \mathrm{mg} / \mathrm{dL})($ level 2$)$, respectively.

\section{Statistical analyses}

Glucose outcomes were calculated with GStat Version 2.2 software (University of Cambridge). Statistical analyses were conducted using SPSS, R, and Stata version 14.1. The percentage of time in target, time of hypoglycemia and time of hyperglycemia were summarized as mean (standard deviation [SD]) or medians (interquartile range [IQR]) where appropriate. Student's $t$-tests and Mann-Whitney-Wilcoxon tests were performed as appropriate and presented with $P$-values and/or $95 \%$ confidence intervals. A $P$-value of $<0.05$ was considered significant.

\section{Results}

Of the 32 women enrolled in the two closed-loop trials, 27 $(84.4 \%)$ used closed loop during labor and delivery. They had a mean age (SD) of 32.6 (4.6) years, diabetes duration of 21.9 (8.9) years, booking HbA1c 7.4 (0.9)\% (57.2 [10.3] mmol/ $\mathrm{mol}$ ), and body mass index of $28.3(4.7) \mathrm{kg} / \mathrm{m}^{2}$. Five women did not use closed loop during labor and delivery $(n=2$ for overnight trial and $n=3$ for day-and-night trial). The reasons for this included: maintaining tight glucose control on SAP $(n=2)$, intermittent use $(n=1)$, delivery out of the country $(n=1)$, and unknown $(n=1)$.

\section{Anesthesia and infant delivery}

The closed-loop system achieved a median time in target of 84.3 (IQR 74.7, 88.8)\%, 84.4 (IQR 48.5, 93.7)\%, and 76.5 
Table 1. Glucose Control During Labor and Delivery by Mode of Delivery

\begin{tabular}{lccc}
\hline & $\begin{array}{c}\text { Vaginal } \\
\text { delivery } \\
\mathrm{n}=4\end{array}$ & $\begin{array}{c}\text { Emergency } \\
\text { cesarean } \\
\text { section } \mathrm{n}=12\end{array}$ & $\begin{array}{c}\text { Elective } \\
\text { cesarean } \\
\text { section } \mathrm{n}=11\end{array}$ \\
\hline Mean glucose, mmol/L (SD) & $6.3(0.2)$ & $7.0(1.5)$ & $7.0(1.6)$ \\
Mean glucose, mg/dL (SD) & $113(3.6)$ & $126(27)$ & $126(29)$ \\
Median time-in-target*, \% (IQR) & $84.3(74.7,88.8)$ & $84.4(48.5,93.7)$ & $76.5(48.2,93.0)$ \\
Median time below target, \% (IQR) & $0(0,3.4)$ & $0.8(0,2.0)$ & $0(0,2.2)$ \\
Median time above target, \% (IQR) & $15.7(11.2,22.0)$ & $11.4(6.3,50.4)$ & $16.5(7.1,51.8)$ \\
Median sensor glucose SD, mmol/L (IQR) & $1.7(1.4,2.0)$ & $1.5(1.2,2.2)$ & $1.8(1.1,2.2)$ \\
Median sensor glucose SD, mg/dL (IQR) & $31(25,36)$ & $27(22,40)$ & $32(20,40)$ \\
Median number of hypoglycemic events (IQR) & $0(0,1.0)$ & $0(0,1.0)$ & $0(0,1.0)$ \\
Number of women with a hypoglycemic event $(\%)$ & $1(25.0)$ & $3(27.2) n=11$ & $3(27.2)$ \\
\hline
\end{tabular}

*The glucose target range during labor and delivery was $3.5-7.8 \mathrm{mmol} / \mathrm{L}(63-140 \mathrm{mg} / \mathrm{dL})$.

$\mathrm{IQR}$, interquartile range; $\mathrm{SD}$, standard deviation.

(IQR 48.2, 93.0)\% for vaginal delivery, emergency cesarean section, and elective cesarean section, respectively (Table 1). Participants also used closed loop under a variety of different methods of anesthesia, including spinal, epidural, and general anesthetic. Closed loop was used during both monopolar and bipolar diathermy without complication. Monopolar and bipolar diathermy are tools commonly used in surgery, including cesarean section, during which electric current is used to cut tissues or achieve hemostasis. Additionally, one participant underwent abdominal cystectomy under general anesthetic following cesarean section under a spinal anesthetic. She maintained near normoglycemia throughout.

\section{Obstetrical outcomes}

The median gestational age at delivery was 37.0 weeks (IQR 34.6, 37.7), with eight preterm deliveries $(29.6 \%)$ and $15(55.6 \%)$ large-for-gestational-age infants. There were 18 neonates $(66.7 \%)$ with hypoglycemia defined as a glucose concentration of $<2.6 \mathrm{mmol} / \mathrm{L}(47 \mathrm{mg} / \mathrm{dL})$; of these, 15 infants $(56 \%)$ were admitted to a neonatal intensive care unit (NICU). A further three infants were admitted to NICU for treatment of sepsis or jaundice but did not have neonatal

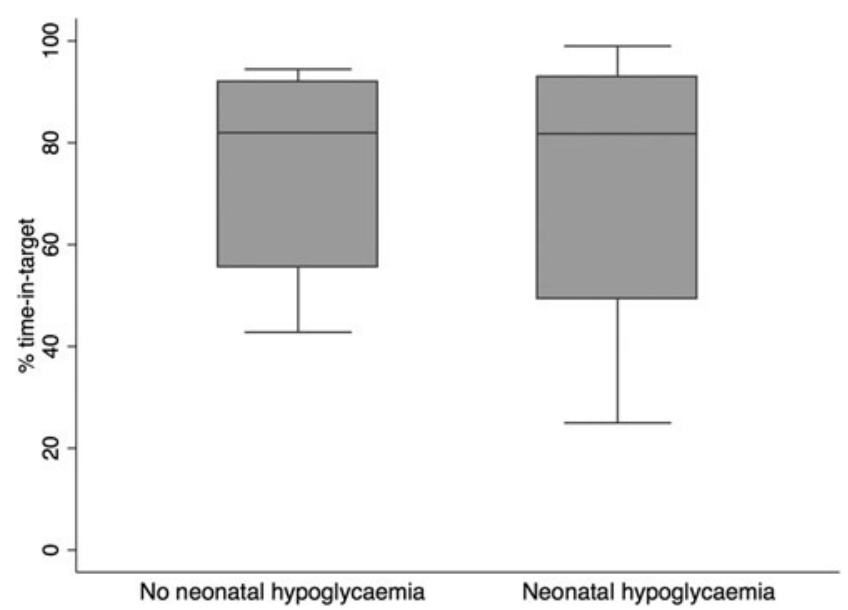

FIG. 1. Box plot of percentage of time in target during labor and delivery for mothers of neonates with and without neonatal hypoglycemia. hypoglycemia. Six women had preeclampsia, three had postpartum hemorrhages, and one had a severe urinary tract infection requiring intravenous antibiotics.

\section{Glycemic control outcomes}

Women who used closed loop during labor and delivery were in the target glucose range 82.0 (IQR 49.3, 93.0)\% of time, with a mean glucose concentration of $6.9(1.4) \mathrm{mmol} / \mathrm{L}$ (124 [25] mg/dL). Median sensor glucose variability measured by SD was $1.7(1.2,2.1) \mathrm{mmol} / \mathrm{L}(31[22,38] \mathrm{mg} / \mathrm{dL})$. The time spent above target was 16.0 (IQR 7.1, 46.7)\%. Hypoglycemia was uncommon; median (IQR) time was below target $0(0,2.2) \%$. The median (IQR) number of hypoglycemic events was $0(0,1.0)$, although seven women $(26.9 \%)$ had at least 1 hypoglycemic event.

In the first $48 \mathrm{~h}$ postpartum, the mean glucose was 7.2 (1.4) $\mathrm{mmol} / \mathrm{L}(130$ [25] $\mathrm{mg} / \mathrm{dL})$. During this period, women spent 83.3 (IQR 75.2, 94.6)\% of the time-in-target range, with minimal hyperglycemia median of (IQR) $9.1(1.5,20.6) \%$. Median sensor glucose variability measured by SD was 2.4 $(1.7,2.8) \mathrm{mmol} / \mathrm{L}(43[31,50] \mathrm{mg} / \mathrm{dL})$. Mild (level 1) hypoglycemia was slightly more common postpartum than during labor and delivery; median (IQR) time $<3.9 \mathrm{mmol} / \mathrm{L}$ $(<70 \mathrm{mg} / \mathrm{dL})$ was $2.5(0.9,5.8) \%$. The median (IQR) number of hypoglycemic events was $1.5(1.0,3.0)$. If a threshold (level 2 hypoglycemia) of $<3.5 \mathrm{mmol} / \mathrm{L}(<63 \mathrm{mg} / \mathrm{dL})$ was used, women did not have any hypoglycemic events in the immediate postpartum period.

There were no incidents of severe hypoglycemia during labor, delivery, or the immediate postpartum period, nor were there serious device-related adverse events during this time.

\section{Neonatal hypoglycemia and maternal glucose concentration in labor}

There was no difference in mean glucose in mothers of infants with neonatal hypoglycemia and those without (mean [SD] maternal glucose of 6.9 [1.6] $\mathrm{mmol} / \mathrm{L}$ and 6.8 [1.1] $\mathrm{mmol} / \mathrm{L}$ [124 (29) and 122 (20) $\mathrm{mg} / \mathrm{dL}]$, respectively; $P=0.84)$. There was also no difference in the percentage of time in target $(P=0.88)$ (Fig. 1) or time above target $(P=0.76)$. 


\section{Conclusions}

In this cohort of women with a long duration of T1D, closed loop performed well during labor and delivery. Women spent over $80 \%$ time-in-target range with minimal hypoglycemia. Closed loop maintained tight glycemic control during vaginal deliveries and cesarean section under both regional and general anesthesia. The system also adapted quickly to the rapid change in insulin requirements after delivery with women spending over $80 \%$ time-intarget range in the immediate postpartum period.

The mean glucose achieved by women using closed loop was comparable to that achieved by other cohorts examining glycemic control in labor, allowing for differences in definitions and glycemic targets. ${ }^{7,12,13}$ Limited data are available when evaluating time in target specifically. Drever et al. reported time in target during labor and delivery using capillary glucose monitoring in a retrospective observational study of T1D women managed on VRIII or CSII. ${ }^{7}$ They found a mean (SD) overall time in target of 47.7 (34.9)\%. They had a tight glucose control target range $(4.0-6.0 \mathrm{mmol} / \mathrm{L}$ [72$108 \mathrm{mg} / \mathrm{dL}]$ ) with almost one third of women experiencing at least one episode of hypoglycemia. They also found that the mean maternal glucose concentration during delivery was unrelated to poor neonatal outcomes, including neonatal hypoglycemia.

Closed-loop insulin delivery also offers potential benefits beyond achieving optimal glycemic control. It may reduce the need to transition from subcutaneous insulin therapy to VRIII, which is expensive, invasive, limits mobility, and can be distressing to women in labor. In addition to limited evidence regarding its clinical efficacy, VRIII places a heavy burden on the obstetric and delivery unit staff, who may have limited diabetes management experience.

The rapid reduction in insulin requirements postpartum is difficult to predict and highly variable, ${ }^{10}$ so closed loop might be particularly useful in adjusting insulin doses in the postpartum period. Closed loop has been demonstrated to be feasible and effective in general and critical care inpatient settings ${ }^{14,15}$ and offers the potential to improve inpatient glycemic control while reducing the burden on staff, many of whom are not specialized in diabetes management. In a randomized controlled trial of 40 nonpregnant inpatients with type 2 diabetes, a fully closed-loop system was associated with a $22 \%$ improvement in the time-in-target range, compared with the routine hospital protocol and was generally well-received by ward staff. ${ }^{14}$ These participants were unfamiliar with CGM or insulin pumps before hospital admission.

This demonstrates the potential for closed loop to be used for isolated short-term periods when achieving optimal glycemic control is challenging, including among diabetes technology-naive participants. During pregnancy, these periods might include antenatal steroid administration, labor, and delivery. It is important, however, to recognize that if this approach were to be adopted, obstetric and delivery ward staff would need to be trained in the basic principles of closed-loop use and specialized support staff would need to be available in case of technical difficulties. ${ }^{16}$

The main limitation of our study is the lack of a standard VRIII or CSII control group with which to compare glucose control. This was an observational study that included wo- men who chose to use closed loop following the randomized portion of two trials. This may have introduced bias in that women included may be different from those who chose not to use closed loop in labor and delivery. Unfortunately, we did not have detailed information about postpartum insulin dosing in the second trial, so these data could not be presented. We also lack data regarding the acceptability of the system to hospital staff, although participants themselves expressed generally high levels of satisfaction regarding closed-loop experiences in semistructured interviews conducted in related psychosocial studies. ${ }^{8}$ Thematic analysis of these interviews revealed no negative comments regarding participants' experiences of closed loop. Participants' positive comments included: "it just took all the worry away, to be honest" (Participant 15, day-and-night trial); "there was me saying to all these doctors, no I'd rather stay on that [closed-loop], not the sliding scale..." (Participant 8, overnight trial); and "I was using it even in my labor, my own closed-loop, because it was working fine in making me more relaxed... so I chose that [sic], rather than choosing the scale, the insulin scale, because I felt more confident with the closed-loop" (Participant 11, day-and-night trial).

Our study demonstrates the adaptability of closed loop during labor, delivery, and the immediate postpartum period. Further research is needed to compare the efficacy of closed loop with current intrapartum sliding scale approaches and to better understand the complex relationship between intrapartum glucose and neonatal hypoglycemia. As well, larger qualitative studies should be performed to learn more about the perception of women with diabetes and their healthcare providers regarding the acceptability of closedloop insulin delivery in this setting.

\section{Acknowledgments}

ZAS, HRM, MEW, and RH conceived of and designed the study. RH designed the control algorithm. ZAS, SH, and HRM participated in data collection. ZAS, JMY, and HRM analyzed and/or interpreted data. CF conducted and analyzed qualitative interviews for related psychosocial studies. JMY and ZAS prepared the article, which all authors critically reviewed. All authors have given final approval of the version to be published. ZAS is the guarantor of this, had full access to all the study data, and takes responsibility for the integrity of the data. The trials were funded by the National Institute for Health Research (HRM Career Development Fellowship, CDF-2013-06-035), Diabetes UK (BDA 07/0003551), Gates Cambridge Trust PhD fellowship (ZAS), Jean Hailes for Women's Health (ZAS); Allen-Carey Scholarship in Women's Health (JMY) and a grant from the NIHR Cambridge Biomedical Research Center (RH). Abbott Diabetes Care supplied discounted CGM devices, sensors, and details of communication protocol to facilitate real-time connectivity. HRM conducts independent research supported by the National Institute for Health Research. The views expressed in this publication are those of the authors and not necessarily those of the NHS, the National Institute for Health Research or the UK Department of Health.

\section{Author Disclosure Statement}

HRM serves on the Medtronic European Scientific Advisory Board. RH received speaker honoraria from Eli Lilly 
and Novo Nordisk and license fees from B Braun and Medtronic; is on advisory panels for Eli Lilly and Novo Nordisk; has served as a consultant to B Braun; and reports patents and patent applications. MEW received license fees from Becton Dickinson, has served as a consultant to Beckton Dickinson, and reports patents and patent applications.

\section{References}

1. Murphy HR, Bell R, Cartwright C, et al.: Improved pregnancy outcomes in women with type 1 and type 2 diabetes but substantial clinic-to-clinic variations: a prospective nationwide study. Diabetologia 2017;60:1668-1677.

2. Yamamoto JM, Benham J, Mohammad K, et al.: Intrapartum glycaemic control and neonatal hypoglycaemia in pregnancies complicated by diabetes: a systematic review. Diabet Med 2018;35:173-183.

3. Canadian Diabetes Association Clinical Practice Guidelines Expert Committee, Thompson D, Berger H, et al.: Diabetes and pregnancy. Can J Diabetes 2013;37 Suppl 1: S168-S183.

4. National Institute for Health and Clinical Excellence: Diabetes in pregnancy: management from preconception to the postnatal period https://www.nice.org.uk/guidance/ng3 Accessed February 12, 2018.

5. Dashora U, Temple R, Murphy H: Joint British Diabetes Societies for Inpatient Care: management of glycaemic control in pregnant women with diabetes on obstetric wards and delivery units. https://www.diabetes.org.uk/resourcess3/2017-10/JBDS\%20Pregnancy\%202017\%2020.10.17_0.pdf Accessed February 12, 2018.

6. Maheux PC, Bonin B, Dizazo A, et al.: Glucose homeostasis during spontaneous labor in normal human pregnancy. J Clin Endocrinol Metab 1996;81:209-215.

7. Drever E, Tomlinson G, Bai AD, Feig DS: Insulin pump use compared with intravenous insulin during labour and delivery: the INSPIRED observational cohort study. Diabet Med 2016;33:1253-1259.

8. Farrington C, Stewart ZA, Barnard K, et al.: Experiences of closed-loop insulin delivery among pregnant women with Type 1 diabetes. Diabet Med 2017;34:1461-1469.
9. Berg M, Sparud-Lundin C: Experiences of professional support during pregnancy and childbirth-a qualitative study of women with type 1 diabetes. BMC Pregnancy Childbirth 2009;9:27.

10. Stewart ZA, Wilinska ME, Hartnell S, et al.: Closed-loop insulin delivery during pregnancy in women with type 1 diabetes. N Engl J Med 2016;375:644-654.

11. Stewart ZA, Wilinska ME, Hartnell S, et al.: Day-and-night closed-loop insulin delivery in a broad population of pregnant women with type 1 diabetes: a randomized controlled crossover trial. Diabetes Care 2018 [Epub ahead of print].

12. Cordua S, Secher AL, Ringholm L, et al.: Real-time continuous glucose monitoring during labour and delivery in women with type 1 diabetes-observations from a randomized controlled trial. Diabet Med 2013;30:1374-1381.

13. Fresa R, Visalli N, Di Blasi V, et al.: Experiences of continuous subcutaneous insulin infusion in pregnant women with type 1 diabetes during delivery from four Italian centers: a retrospective observational study. Diabetes Technol Ther 2013;15:328-334.

14. Thabit H, Hartnell S, Allen JM, et al.: Closed-loop insulin delivery in inpatients with type 2 diabetes: a randomised, parallel-group trial. Lancet Diabetes Endocrinol 2017;5: 117-124.

15. Leelarathna L, English SW, Thabit H, et al.: Feasibility of fully automated closed-loop glucose control using continuous subcutaneous glucose measurements in critical illness: a randomized controlled trial. Crit Care 2013;17:R159.

16. Rayman G: Closer to closing the loop on inpatient glycaemia. Lancet Diabetes Endocrinol 2017;5:81-83.

Address correspondence to:

Helen R. Murphy, MD

Department of Medicine, Norwich Medical School University of East Anglia

Floor 2, Bob Champion Research and Education Building

Norwich NR4 7UQ

United Kingdom

E-mail: hm386@medschl.cam.ac.uk 